\title{
Preventions and Controls on Congenital Transmissions of Zika: Mathematical Analysis
}

\author{
Nita H. Shah ${ }^{1}$, Zalak A. Patel ${ }^{2}$, Bijal M. Yeolekar ${ }^{1}$ \\ ${ }^{1}$ Department of Mathematics, Gujarat University, Ahmedabad, Gujarat, India \\ ${ }^{2}$ L. D. College of Engineering, Ahmedabad, Gujarat, India \\ Email: *nitahshah@gmail.com, zalak23patel@gmail.com, bijalyeolekar28@gmail.com
}

How to cite this paper: Shah, N.H., Patel, Z.A. and Yeolekar, B.M. (2017) Preventions and Controls on Congenital Transmissions of Zika: Mathematical Analysis. Applied Mathematics, 8, 500-519.

https://doi.org/10.4236/am.2017.84040

Received: March 16, 2017

Accepted: April 21, 2017

Published: April 24, 2017

Copyright (c) 2017 by authors and Scientific Research Publishing Inc. This work is licensed under the Creative Commons Attribution International License (CC BY 4.0).

http://creativecommons.org/licenses/by/4.0/

(c) (i) Open Access

\begin{abstract}
Vector-borne diseases threat lives of millions of people in many countries of the world. Zika is one of the vector-borne diseases which initially spread by the bite of an infected Aedes species mosquito (Ae. aegypti and Ae. albopictus) and then it transmits vertically from a pregnant woman to her fetus or from an infected human to their sexual partners. The congenital transmission of Zika virus (ZIKV) results in new born with microcephaly and other neurological abnormalities. The control of infected mosquitos is the best efficient way to control spread of ZIKV. Spraying insecticide is the safest and easiest way to control mosquitos, but sometimes it is cost worthy for long period of spraying. Controlled prevention from the vector bites can also help to control disease spread. To control congenital transmission and sexual transmission of ZIKV, preventions should be taken to reduce/stop pregnancy rate and safe heterosexual transmission among adults. Also, there is no specific treatment available for Zika disease. Treatment is aimed at relieving symptoms with rest, fluids and medications. Controlled combinations of rest, fluids and medications will help to recover early. As costs are incorporated with spraying, preventions and treatment, our aim is to minimise the total cost associated by controlling spraying, preventions and treatment. To fulfil this purpose a mathematical model is developed with disease dynamics in nine compartments namely Susceptible human child, Susceptible human male, Susceptible human female, Infected human child, Infected human male, Infected human female, Recovered human, Susceptible vector and Infected vector including vertical transmission of Zika disease. Numerical simulations have been carried out to optimise controls, and basic reproduction number and stability are calculated.
\end{abstract}

\section{Keywords}

Zika, Vertical Transmission, Dynamical System, Control, Stability 


\section{Introduction}

Zika virus (ZIKV) is a Flavivirus. It is initially transmitted to humans by the bites of infected female mosquitoes from the Aedes genus. The pathogen responsible for spread of Zika disease is known as Zika virus (ZIKV).In past two years remarkable changes has been seen in the epidemiology of Zika virus (ZIKV). The transmission of ZIKV has been first reported from continental America and the Caribbean. Also, recent reports indicate an increase in detected cases of congenital malformations and neurological complications associated with ZIKV infection. To treat, prevent, or diagnose ZIKV infection there is no specific treatment, vaccine, or fast diagnostic test available at this time.

Dick et al. [1], Rodrigez et al. [2] and Macnamara et al. [3] observed that in the Zika forest of Uganda the Zika virus was initially isolated from a rhesus monkey in 1947. In 1954 same virus was isolated from humans in Nigeria. There after only sporadic infected human cases were reported from Africa and Southeast Asia. Duffy et al. [4] observed that first largest outbreak of ZIKV infection was reported in 2007 on Yap Island of the North Pacific. Musso et al. [5] observed that, in French Polynesia during October 2013, 28000 ZIKV infection cases were reported. Pan American Health Organization [6] noted that the recent outbreak began in April 2015 from Brazil, has covered many southern, central American countries and the Caribbean with spread of disease, and more than 140,000 suspected and confirmed cases are reported by the end of February 2016.

Foy et al. [7] reported that infection of ZIKV spread from an infected male to a female during their sexual intercourse. Thereafter, in February 2016 cases of sexually transmitted ZIKV were reported from Dallas [8] County, U.S. and France by CDC [9], Hills et al. [10] and Mansuy et al. [11] respectively. According to the Toronto Star [12] spread of Zika infection because of sexual activities has been reported in Argentina, Canada, Chile, France, Italy, New Zealand, Peru, Portugal, and the USA from 2015. Hills et al. [10] recorded that the disease has minor impact on sexual activity as ZIKV infections have mild symptoms for two to seven days. As ZIKV is sexually transmissible, CDC [9] issued related guidelines for preventions should be taken for safe sex during a Zika outbreak. Didier et al. [13] noted that ZIKV has been found in semen samples which point out the possibility of transmission of ZIKV through sexual activity.

Gatherer et al. [14] found that like other flaviviruses ZIKV could also be transmitted by blood transfusion.

Mlakar et al. [15] and Cauchemez et al. [16] indicated that ZIKV increases the chances of microcephaly in new-born babies of infected mothers. Cao-Lormeau et al. [17] derived that it causes Guillain-Barre syndrome (GBS) as well. According to Pan American Health Organization, from October 2015 to February 2016 approximately 6000 suspected cases with 139 deaths of microcephaly in newborns due to ZIKV infection were reported in Brazil, and from December 2015 to February 2016, more than 200 cases and 118 cases with 5 deaths of GBS due to suspected ZIKV infection were recorded in Colombia and in El Salvador respec- 
tively. As per estimation of WHO [18], based on the reported dengue data from 2015, about four million people in the Americas may be infected by ZIKV in 2016. Oliveira et al. [19] observed that in February 2015, abnormal brain development in the foetuses of pregnant and ZIKV infected women were found.

Dalia et al. [20] suggested for prevent pregnancy and effective strategies against the vector will control disease spread. Ebenezer et al. [21] suggested that to control disease the best strategy is to combine all preventive, treatment and Insecticides controls simultaneously. Daozhou et al. [22] noted that to control spread of ZIKV preventive steps are necessary during sexual activities in ZIKV outbreak area.

Together all these facts lead to the increase of the people susceptible to the disease. As there is no vaccination available for the ZIKV disease, it is consider as a severe problem. Mathematical models are essential tools to study the dynamics of the spread of infectious diseases like ZIKV. Basic reproduction number provides information about how infection will be sustained. In this study, a new model with optimal control on spraying insecticides, preventions and treatment is examined. Pontryagin's maximum principle, established by Pontrayagin et al. [23] is used to determine the optimal control. Result proves that optimal control gives significant reduction in ZIKV spread.

The paper is organised as follow. In Section 2, mathematical model by a system of ordinary differential equations with notations, assumptions and the flow of populations between compartments are described. For autonomous model having fixed rates of controls basic reproduction number of the whole system (human-mosquito combined) is calculated at disease free equilibrium and endemic equilibrium points. In Section 3, Stability of model has been discussed. In Section 4, cost control function for controls on spraying, preventions and treatment is formed and validated for the system of equations obtained in Section 2. In Section 5, numerical simulations are carried out, for both autonomous and control model. In Section 6, conclusions suggest that how to control disease spread with minimal cost.

\section{Mathematical Model}

The mathematical model is developed with following notations.

A system of non-linear differential equations is formulated to investigate spread of congenital transmission of ZIKV with control spraying, preventions and treatment. Dynamics of human population $N_{H}(t)$ and Vector population $N_{V}(t)$ are developed. Human population is classified amongst eight compartments viz. number of susceptible human child $S_{H C}(t)$, number of susceptible human female $S_{H F}(t)$, number of susceptible human male $S_{H M}(t)$, number of infected human child $I_{H C}(t)$, number of infected human female $I_{H F}(t)$, number of infected human male $I_{H M}(t)$, number of recovered human adult $R_{H}(t)$, while, vector population $N_{V}(t)$ is divided in to two compartments viz. number of susceptible vectors $S_{V}(t)$, number of infected vectors $I_{V}(t)$. The population dynamics of above compartments is shown in Figure 1. 

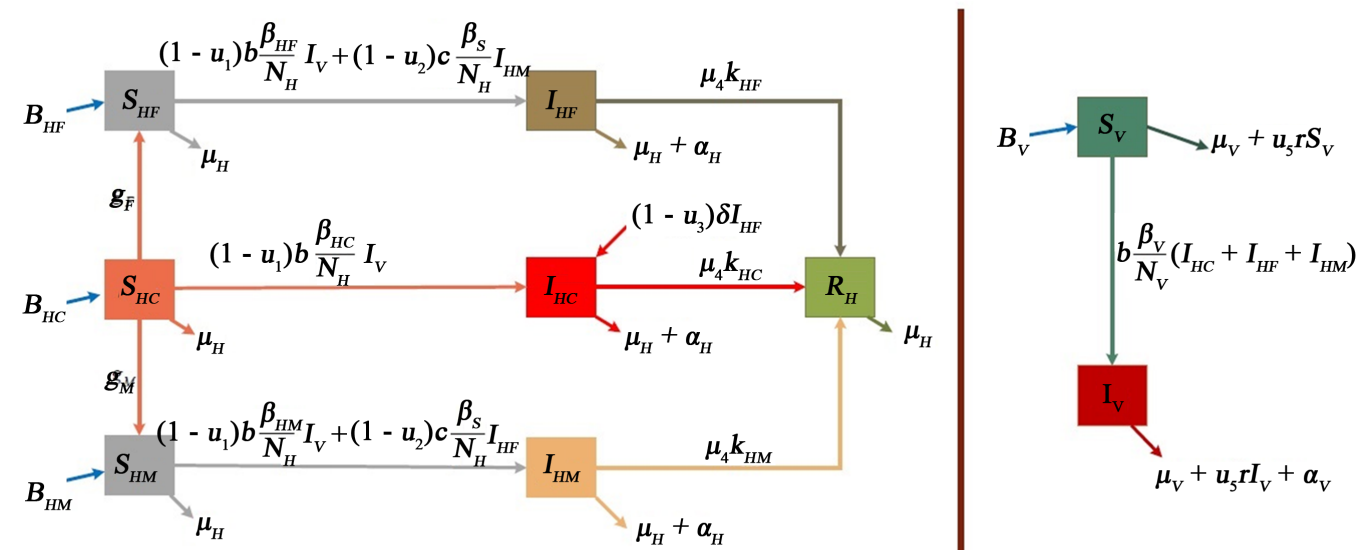

Figure 1. Dynamics of individuals in different compartments.

To investigate effects of congenital transmission of disease and its control by spraying on mosquitoes, preventions and treatment, human population is distributed in human child and human male (adult) and human female (adult) classes.

For model formulation increase in total population of vectors (mosquitoes) is considered, as subpopulation of vectors (mosquitoes) survive from the spraying, get matures and reproduces.

To prepare model, following possibilities of disease spread are considered:

1) Vertical Transmission of infection to new-borns from infected mothers;

2) Horizontal transmission to human child/adult (male and female) from infected vectors (mosquitoes) when they bite to human child/adult (male and female);

3) Heterosexual transmissions amongst human adults (male and female);

4) Horizontal transmission to vectors (mosquitoes) from infected human child/adult (male and female) when vectors (mosquitoes) bite to human child/ adult (male and female).

\subsection{Disease Dynamics amongst Human Population}

After birth from an infected human female, infected human children will join infected human child class at rate $\left(1-u_{3}\right) \delta I_{H F}$. Susceptible human children will either join infected human child class at rate $\left(1-u_{1}\right) b \frac{\beta_{H C}}{N_{H}} I_{V}$ due to bite of an infected vector (mosquito) or susceptible human male (adult) or female (adult) classes at growth rates $g_{M}$ and $g_{F}$ respectively. Infected human child will join recovered human class at recovery/treatment rate $u_{4} k_{H C}$. Disease induced death rate $\alpha_{H C}$ will make very high effect on infected child population as possibility of microcephaly and Guillain-Barre syndrome due to ZIKV transmission occurred during pregnancy. 
Susceptible human male (adult) and female (adult) will either join infected human male (adult) and female (adult) class at rates

$$
\left(1-u_{1}\right) b \frac{\beta_{H M}}{N_{H}} I_{V}+\left(1-u_{2}\right) c \frac{\beta_{S}}{N_{H}} I_{H F} \quad \text { an } \mathrm{d} \quad\left(1-u_{1}\right) b \frac{\beta_{H F}}{N_{H}} I_{V}+\left(1-u_{2}\right) c \frac{\beta_{S}}{N_{H}} I_{H M}
$$

respectively due to vector bite and sexual intercourse with infected human female (adult) and male (adult) respectively. Infected human male (adult) and female (adult) will join recovered human class at recovery/treatment rate $u_{4} k_{H M}$ and $u_{4} k_{H F}$ respectively. Disease induced death rate $\alpha_{H}$ will effect on infected human male and female (adult) population. At each stage, the natural death rate in human child/adult classes $\mu_{H}$ is taken into account.

\subsection{Disease Dynamics amongst Vector (Mosquito) Population}

Vector (mosquito) population is divided in to two compartments susceptible vectors and infected vectors. Susceptible vector gets infection from infected human child/adult, when vectors (mosquitoes) bite them, and joins infected vector class at rate $b \frac{\beta_{V}}{N_{V}}\left(I_{H C}+I_{H F}+I_{H M}\right)$. A portion of susceptible and infected vectors (mosquitoes) will be eliminated due to either natural death rate in vectors (mosquitoes) $\mu_{V}$ or spraying at rate $u_{5} r$. Disease induced death rate $\alpha_{V}$ will also affect the infected vector (mosquito) population.

With above discussion the dynamics of Zika disease can be represented by the system of non-linear differential equations as

$$
\frac{\mathrm{d} S_{H C}(t)}{\mathrm{d} t}=B_{H C}-\left(1-u_{1}\right) b \frac{\beta_{H C}}{N_{H}} I_{V} S_{H C}-\mu_{H} S_{H C}-\left(g_{F}+g_{M}\right) S_{H C}
$$

$$
\frac{\mathrm{d} S_{H F}(t)}{\mathrm{d} t}=B_{H F}-\left(\left(1-u_{1}\right) b \frac{\beta_{H F}}{N_{H}} I_{V}+\left(1-u_{2}\right) c \frac{\beta_{S}}{N_{H}} I_{H M}\right) S_{H F}+g_{F} S_{H C}-\mu_{H} S_{H F}
$$

$\frac{\mathrm{d} S_{H M}(t)}{\mathrm{d} t}=B_{H M}-\left(\left(1-u_{1}\right) b \frac{\beta_{H M}}{N_{H}} I_{V}+\left(1-u_{2}\right) c \frac{\beta_{S}}{N_{H}} I_{H F}\right) S_{H M}+g_{M} S_{H C}-\mu_{H} S_{H M}$

$$
\frac{\mathrm{d} I_{H C}(t)}{\mathrm{d} t}=\left(1-u_{1}\right) b \frac{\beta_{H C}}{N_{H}} I_{V} S_{H C}+\left(1-u_{3}\right) \delta I_{H F}-u_{4} k_{H C} I_{H C}-\left(\mu_{H}+\alpha_{H C}\right) I_{H C}
$$

$$
\frac{\mathrm{d} I_{H F}(t)}{\mathrm{d} t}=\left(\left(1-u_{1}\right) b \frac{\beta_{H F}}{N_{H}} I_{V}+\left(1-u_{2}\right) c \frac{\beta_{S}}{N_{H}} I_{H M}\right) S_{H F}-u_{4} k_{H F} I_{H F}-\left(\mu_{H}+\alpha_{H}\right) I_{H F}
$$

$$
\begin{gathered}
\frac{\mathrm{d} I_{H M}(t)}{\mathrm{d} t}=\left(\left(1-u_{1}\right) b \frac{\beta_{H M}}{N_{H}} I_{V}+\left(1-u_{2}\right) c \frac{\beta_{S}}{N_{H}} I_{H F}\right) S_{H M}-u_{4} k_{H M} I_{H M}-\left(\mu_{H}+\alpha_{H}\right) I_{H M} \\
\frac{\mathrm{d} R_{H}(t)}{\mathrm{d} t}=u_{4}\left(k_{H C} I_{H C}+k_{H F} I_{H F}+k_{H M} I_{H M}\right)-\mu_{H} R_{H} \\
\frac{\mathrm{d} S_{V}(t)}{\mathrm{d} t}=B_{V}-\mu_{V} S_{V}-b \frac{\beta_{V}}{N_{V}}\left(I_{H C}+I_{H F}+I_{H M}\right) S_{V}-u_{5} r S_{V} \\
\frac{\mathrm{d} I_{V}(t)}{\mathrm{d} t}=b \frac{\beta_{V}}{N_{V}}\left(I_{H C}+I_{H F}+I_{H M}\right) S_{V}-\left(\mu_{V}+\alpha_{V}\right) I_{V}-u_{5} r I_{V}
\end{gathered}
$$

Adding Equations (1) to (7), 


$$
\begin{aligned}
\frac{\mathrm{d} N_{H}}{\mathrm{~d} t}= & B_{H C}+B_{H F}+B_{H M}-\mu_{H}\left(S_{H C}+S_{H F}+S_{H M}+I_{H C}+I_{H F}+I_{H M}+R_{H}\right) \\
& +\left(1-u_{3}\right) \delta I_{H F}-\alpha_{H C} I_{H C}-\alpha_{H}\left(I_{H F}+I_{H M}\right)
\end{aligned}
$$

And, adding Equations (8) and (9),

$$
\frac{\mathrm{d} N_{V}}{\mathrm{~d} t}=B_{V}-\mu_{V}\left(S_{V}+I_{V}\right)-u_{5} r\left(S_{V}+I_{V}\right)-\alpha_{V} I_{V}
$$

Hence,

$$
\frac{\mathrm{d} N_{H}}{\mathrm{~d} t} \leq B_{H C}+B_{H F}+B_{H M}-\mu_{H} N_{H} \Rightarrow \lim _{t \rightarrow \infty} \operatorname{Sup}_{H} \leq \frac{B_{H C}+B_{H F}+B_{H M}}{\mu_{H}}
$$

and,

$$
\frac{\mathrm{d} N_{V}}{\mathrm{~d} t} \leq B_{V}-\mu_{V} N_{V} \Rightarrow \lim _{t \rightarrow \infty} \operatorname{Sup}_{V} \leq \frac{B_{V}}{\mu_{V}} .
$$

So, the feasible region of the system is

$$
\Omega=\left\{\begin{array}{c}
\left(S_{H C}, S_{H F}, S_{H M}, I_{H C}, I_{H F}, I_{H M}, R_{H}, S_{V}, I_{V}\right): \\
S_{T H C}+S_{U H C}+S_{T H A}+S_{U H A}+I_{T H C}+I_{U H C}+I_{T H A}+I_{U H A} \leq \frac{B_{H C}+B_{H F}+B_{H M}}{\mu_{H}}, \\
S_{V}+I_{V} \leq \frac{B_{V}}{\mu_{V}}, \\
S_{H C}>0, S_{H F}>0, S_{H M}>0, I_{H C}>0, I_{H F} \geq 0, I_{H M} \geq 0, R_{H} \geq 0, S_{V}>0, I_{V} \geq 0
\end{array}\right\}
$$

\subsection{Computation of Basic Reproduction Number $R_{0}$}

As at disease free equilibrium, $I_{H C}=0, I_{H F}=0, I_{H M}=0, I_{V}=0$ and $R_{H}=0$.

Hence, let $X_{0}=\left(\frac{B_{H C}}{\mu_{H}+g_{F}+g_{M}}, \frac{B_{H F}}{\mu_{H}-g_{F}}, \frac{B_{H M}}{\mu_{H}-g_{M}}, 0,0,0,0, \frac{B_{V}}{\mu_{V}+u_{5} r}, 0\right)$ be disease free equilibrium point of system.

Using next generation method [24], let

$$
X^{\prime}=\left(I_{H C}, I_{H F}, I_{H M}, I_{V}, S_{H C}, S_{H F}, S_{H M}, S_{V}, R_{H}\right)^{\prime}
$$

where dash denotes derivative

$$
\therefore X^{\prime}=\frac{\mathrm{d} X}{\mathrm{~d} t}=\mathfrak{I}(x)-v(x)
$$

where,

$$
\Im(X)=\left(\begin{array}{c}
\left(1-u_{1}\right) b \frac{\beta_{H C}}{N_{H}} I_{V} S_{H C} \\
\left(1-u_{1}\right) b \frac{\beta_{H F}}{N_{H}} I_{V} S_{H F}+\left(1-u_{2}\right) c \frac{\beta_{S}}{N_{H}} I_{H M} S_{H F} \\
\left(1-u_{1}\right) b \frac{\beta_{H M}}{N_{H}} I_{V} S_{H M}+\left(1-u_{2}\right) c \frac{\beta_{S}}{N_{H}} I_{H F} S_{H M} \\
b \frac{\beta_{V}}{N_{V}}\left(I_{H C}+I_{H F}+I_{H M}\right) S_{V} \\
0 \\
0 \\
0 \\
0 \\
0
\end{array}\right)
$$


and

$$
v(X)=\left(\begin{array}{c}
-\left(1-u_{3}\right) \delta I_{H F}+u_{4} k_{H C} I_{H C}+\mu_{H} I_{H C}+\alpha_{H C} I_{H C} \\
u_{4} k_{H F} I_{H F}+\mu_{H} I_{H F}+\alpha_{H} I_{H F} \\
u_{4} k_{H M} I_{H M}+\mu_{H} I_{H M}+\alpha_{H} I_{H M} \\
\mu_{V} I_{V}+\alpha_{V} I_{V}+u_{5} r I_{V} \\
-B_{H F}+\left(1-u_{1}\right) b \frac{\beta_{H F}}{N_{H}} I_{V} S_{H F}+\left(1-u_{2}\right) c \frac{\beta_{S}}{N_{H}} I_{H M} S_{H F}+\mu_{H} S_{H F}-g_{F} S_{H C} \\
-B_{H C}+\left(1-u_{1}\right) b \frac{\beta_{H C}}{N_{V}} S_{H C}+\mu_{H} S_{H C}+\left(g_{F}+g_{M}\right) S_{H C} \\
-B_{H M}+\left(1-u_{1}\right) b \frac{\beta_{H M}}{N_{H}} I_{V} S_{H M}+\left(1-u_{2}\right) c \frac{\beta_{S}}{N_{H}} I_{H F} S_{H F}+\mu_{H} S_{H M}-g_{M} S_{H C} \\
-B_{V}+b \frac{\beta_{V}}{N_{V}}\left(I_{H C}+I_{H F}+I_{H M}\right) S_{V}+u_{5} r S_{V}+\mu_{V} S_{V} \\
\alpha_{V} S_{V}+\mu_{V} I_{V}+u_{5} r I_{V} \\
\text { Using, } F=\left[\frac{\partial \Im_{i}\left(X_{0}\right)}{\partial X_{j}}\right] \text { and } V=\left[\frac{\partial v_{i}\left(X_{0}\right)}{\partial X_{j}}\right] \text { for } i, j=1,2,3, \cdots, 9
\end{array}\right.
$$

Therefore,

$$
F=\left(\begin{array}{ccccccccc}
0 & 0 & 0 & b_{14} & 0 & 0 & 0 & 0 & 0 \\
0 & 0 & b_{23} & b_{24} & 0 & 0 & 0 & 0 & 0 \\
0 & b_{32} & 0 & b_{34} & 0 & 0 & 0 & 0 & 0 \\
b_{41} & b_{42} & b_{43} & 0 & 0 & 0 & 0 & 0 & 0 \\
0 & 0 & 0 & 0 & 0 & 0 & 0 & 0 & 0 \\
0 & 0 & 0 & 0 & 0 & 0 & 0 & 0 & 0 \\
0 & 0 & 0 & 0 & 0 & 0 & 0 & 0 & 0 \\
0 & 0 & 0 & 0 & 0 & 0 & 0 & 0 & 0 \\
0 & 0 & 0 & 0 & 0 & 0 & 0 & 0 & 0
\end{array}\right)
$$

where,

$$
\begin{aligned}
b_{14} & =\frac{\left(1-u_{1}\right) b \beta_{H C} B_{H C}}{\left(\mu_{H}+g_{F}+g_{M}\right) N_{H}}, & b_{23} & =\frac{\left(1-u_{2}\right) c \beta_{S} B_{H F}}{\left(\mu_{H}-g_{F}\right) N_{H}}, \\
b_{24} & =\frac{\left(1-u_{1}\right) b \beta_{H F} B_{H F}}{\left(\mu_{H}-g_{F}\right) N_{H}}, & b_{32} & =\frac{\left(1-u_{2}\right) c \beta_{S} B_{H M}}{\left(\mu_{H}-g_{M}\right) N_{H}}, \\
b_{34} & =\frac{\left(1-u_{1}\right) b \beta_{H M} B_{H M}}{\left(\mu_{H}-g_{M}\right) N_{H}}, & b_{41} & =\frac{b \beta_{V} B_{V}}{\left(\mu_{V}+u_{5} r\right) N_{V}}, \\
b_{42} & =\frac{b \beta_{V} B_{V}}{\left(\mu_{V}+u_{5} r\right) N_{V}}, & b_{43} & =\frac{b \beta_{V} B_{V}}{\left(\mu_{V}+u_{5} r\right) N_{V}}
\end{aligned}
$$

and

$$
V=\left(\begin{array}{ccccccccc}
a_{11} & a_{12} & 0 & 0 & 0 & 0 & 0 & 0 & 0 \\
0 & a_{22} & 0 & 0 & 0 & 0 & 0 & 0 & 0 \\
0 & 0 & a_{33} & 0 & 0 & 0 & 0 & 0 & 0 \\
0 & 0 & 0 & a_{44} & 0 & 0 & 0 & 0 & 0 \\
0 & 0 & 0 & a_{54} & a_{55} & 0 & 0 & 0 & 0 \\
0 & 0 & a_{63} & a_{64} & 0 & a_{66} & 0 & 0 & 0 \\
0 & a_{72} & 0 & a_{74} & 0 & 0 & a_{77} & 0 & 0 \\
a_{81} & a_{82} & a_{83} & 0 & 0 & 0 & 0 & a_{88} & 0 \\
a_{91} & a_{92} & a_{93} & 0 & 0 & 0 & 0 & 0 & a_{99}
\end{array}\right)
$$


where,

$$
\begin{array}{ll}
a_{11}=u_{4} k_{H C}+\mu_{H}+\alpha_{H C}, & a_{12}=-\left(1-u_{3}\right) \delta, \\
a_{22}=u_{4} k_{H F}+\mu_{H}+\alpha_{H}, & a_{33}=u_{4} k_{H M}+\mu_{H}+\alpha_{H} \\
a_{33}=u_{4} k_{H M}+\mu_{H}+\alpha_{H}, & a_{44}=\mu_{V}+\alpha_{V}+u_{5} r, \\
a_{54}=\frac{\left(1-u_{1}\right) b \beta_{H C} B_{H C}}{\left(\mu_{H}+g_{F}+g_{M}\right) N_{H}}, & a_{55}=\mu_{H}+g_{F}+g_{M}, \\
a_{63}=\frac{\left(1-u_{2}\right) c \beta_{S} B_{H F}}{\left(\mu_{H}-g_{F}\right) N_{H}}, & a_{64}=\frac{\left(1-u_{1}\right) b \beta_{H F} B_{H F}}{\left(\mu_{H}-g_{F}\right) N_{H}}, \\
a_{66}=\mu_{H}-g_{F}, & a_{72}=\frac{\left(1-u_{2}\right) c \beta_{S} B_{H M}}{\left(\mu_{H}-g_{M}\right) N_{H}}, \\
a_{74}=\frac{\left(1-u_{1}\right) b \beta_{H M} B_{H M}}{\left(\mu_{H}-g_{M}\right) N_{H}}, & a_{77}=\mu_{H}-g_{M}, \\
a_{81}=\frac{b \beta_{V} B_{V}}{\left(\mu_{V}+u_{5} r\right) N_{V}}, & a_{82}=\frac{b \beta_{V} B_{V}}{\left(\mu_{V}+u_{5} r\right) N_{V}}, \\
a_{83}=\frac{b \beta_{V} B_{V}}{\left(\mu_{V}+u_{5} r\right) N_{V}}, & a_{88}=\mu_{V}+u_{5} r, \\
a_{91}=-u_{4} k_{H C}, & a_{92}=-u_{4} k_{H F}, \\
a_{93}=-u_{4} k_{H M}, & a_{99}=\mu_{H}
\end{array}
$$

The basic reproduction number $R_{0}$ is spectral radius of matrix $F V^{-1}$. With the parametric values given in Table $1, R_{0}=0.4757$.

\section{Stability}

\subsection{Stability at Disease Free Equilibrium}

If all eigenvalues of Jacobian matrix of the system of differential Equations (1) to (10) have negative real parts at

$$
X_{0}=\left(\frac{B_{H C}}{\mu_{H}+g_{F}+g_{M}}, \frac{B_{H F}}{\mu_{H}-g_{F}}, \frac{B_{H M}}{\mu_{H}-g_{M}}, 0,0,0,0, \frac{B_{V}}{\mu_{V}+u_{5} r}, 0\right)
$$

then disease free equilibrium point becomes stable. Jacobian matrix of the system at DFE is,

$$
J=\left(\begin{array}{ccccccccc}
c_{11} & 0 & 0 & 0 & 0 & 0 & 0 & 0 & c_{19} \\
0 & c_{22} & 0 & 0 & 0 & c_{26} & 0 & 0 & c_{29} \\
0 & 0 & c_{33} & 0 & c_{35} & 0 & 0 & 0 & c_{39} \\
0 & 0 & 0 & c_{44} & c_{45} & 0 & 0 & 0 & c_{49} \\
0 & 0 & 0 & 0 & c_{55} & c_{56} & 0 & 0 & c_{59} \\
0 & 0 & 0 & 0 & c_{65} & c_{66} & 0 & 0 & c_{69} \\
0 & 0 & 0 & c_{74} & c_{75} & c_{76} & c_{77} & 0 & 0 \\
0 & 0 & 0 & c_{84} & c_{85} & c_{86} & 0 & c_{88} & 0 \\
0 & 0 & 0 & c_{94} & c_{95} & c_{96} & 0 & 0 & c_{99}
\end{array}\right)
$$

where, 


$$
\begin{aligned}
& c_{11}=-\mu_{H}-g_{F}-g_{M}, \quad c_{19}=-\frac{\left(1-u_{1}\right) b \beta_{H C} B_{H C}}{\left(\mu_{H}+g_{F}+g_{M}\right) N_{H}}, \\
& c_{21}=g_{F}, \quad c_{22}=-\mu_{H}, \quad c_{26}=-\frac{\left(1-u_{2}\right) c \beta_{S} B_{H F}}{\left(\mu_{H}-g_{F}\right) N_{H}}, \\
& c_{29}=-\frac{\left(1-u_{1}\right) b \beta_{H F} B_{H F}}{\left(\mu_{H}-g_{F}\right) N_{H}}, \quad c_{31}=g_{M}, \quad c_{33}=-\mu_{H}, \\
& c_{35}=-\frac{\left(1-u_{2}\right) c \beta_{S} B_{H M}}{\left(\mu_{H}-g_{M}\right) N_{H}}, \quad c_{39}=-\frac{\left(1-u_{1}\right) b \beta_{H M} B_{H M}}{\left(\mu_{H}-g_{M}\right) N_{H}}, \\
& c_{44}=-u_{4} k_{H C}-\mu_{H}-\alpha_{H C}, \quad c_{45}=\left(1-u_{3}\right) \delta, \\
& c_{49}=-c_{19}, \quad c_{55}=-u_{4} k_{H F}-\mu_{H}-\alpha_{H}, \quad c_{56}=-c_{26}, \quad c_{59}=-c_{29}, \\
& c_{65}=-c_{35}, \quad c_{66}=-u_{4} k_{H M}-\mu_{H}-\alpha_{H}, \quad c_{69}=-c_{39}, \\
& c_{74}=u_{4} k_{H C}, \quad c_{75}=u_{4} k_{H F}, \quad c_{76}=u_{4} k_{H M}, \quad c_{77}=-\mu_{H}, \\
& c_{84}=-\frac{b \beta_{V} B_{V}}{\left(\mu_{V}+u_{5} r\right) N_{V}}, \quad c_{85}=c_{84}, \quad c_{86}=c_{84}, \quad c_{88}=-\mu_{V}-u_{5} r, \\
& c_{94}=-c_{84}, \quad c_{95}=-c_{84}, \quad c_{96}=-c_{84}, \quad c_{99}=c_{88}-\alpha_{V} .
\end{aligned}
$$

With the parametric values given in Table 1 , trace $(J)<0$ and also $\operatorname{det}(J)>0$, if $R_{0}<1$.

This implies that disease free equilibrium is locally asymptotically stable if $R_{0}<1$ and unstable, if $R_{0}>1$.

\subsection{Stability at Endemic Equilibrium Point}

For the endemic equilibrium, using

$$
\begin{array}{ll}
\frac{\mathrm{d} S_{T H C}}{\mathrm{~d} t}=0, & \frac{\mathrm{d} S_{U H C}}{\mathrm{~d} t}=0, \quad \frac{\mathrm{d} S_{T H A}}{\mathrm{~d} t}=0, \\
\frac{\mathrm{d} S_{U H A}}{\mathrm{~d} t}=0, & \frac{\mathrm{d} I_{T H C}}{\mathrm{~d} t}=0, \\
\frac{\mathrm{d} I_{U H C}}{\mathrm{~d} t}=0, & \frac{\mathrm{d} I_{T H A}}{\mathrm{~d} t}=0, \\
\frac{\mathrm{d} I_{U H A}}{\mathrm{~d} t}=0, & \frac{\mathrm{d} S_{V}}{\mathrm{~d} t}=0, \quad \frac{\mathrm{d} I_{V}}{\mathrm{~d} t}=0
\end{array}
$$

These give, $X^{*}=\left(S_{T H C}^{*}, S_{U H C}^{*}, S_{T H A}^{*}, S_{U H A}^{*}, I_{T H C}^{*}, I_{U H C}^{*}, I_{T H A}^{*}, I_{U H A}^{*}, S_{V}^{*}, I_{V}^{*}\right)$ as an endemic equilibrium point.

$$
J^{*}=\left(\begin{array}{ccccccccc}
d_{11} & 0 & 0 & 0 & 0 & 0 & 0 & 0 & d_{19} \\
d_{21} & d_{22} & 0 & 0 & 0 & d_{26} & 0 & 0 & d_{29} \\
d_{31} & 0 & d_{33} & 0 & d_{35} & 0 & 0 & 0 & d_{39} \\
d_{41} & 0 & 0 & d_{44} & d_{45} & 0 & 0 & 0 & d_{49} \\
0 & d_{52} & 0 & 0 & d_{55} & d_{56} & 0 & 0 & d_{59} \\
0 & 0 & d_{63} & 0 & d_{65} & d_{66} & 0 & 0 & d_{69} \\
0 & 0 & 0 & d_{74} & d_{75} & d_{76} & d_{77} & 0 & 0 \\
0 & 0 & 0 & d_{84} & d_{85} & d_{86} & 0 & d_{88} & 0 \\
0 & 0 & 0 & d_{94} & d_{95} & d_{96} & 0 & d_{98} & d_{99}
\end{array}\right)
$$

where, 
Table 1. Description of notation and parametric values.

\begin{tabular}{|c|c|c|}
\hline Notation & Description & Parametric values \\
\hline$N_{H}(t)$ & Human Population at time $t$ & 1600 \\
\hline$N_{V}(t)$ & Vector Population at time $t$ & 20000 \\
\hline$S_{H C}(t)$ & Number of susceptible Human Child at time $t$ & 20 \\
\hline$S_{H F}(t)$ & Number of susceptible Human female at time $t$ & 40 \\
\hline$S_{\text {нм }}(t)$ & Number of susceptible Human male at time $t$ & 30 \\
\hline$I_{\text {HС }}(t)$ & Number of Infected Human Child at time $t$ & 10 \\
\hline$I_{H F}(t)$ & Number of Infected Human female at time $t$ & 20 \\
\hline$I_{H M}(t)$ & Number of Infected Human male at time $t$ & 12 \\
\hline$R_{H}(t)$ & Number of Recovered Human at time $t$ & 80 \\
\hline$S_{v}(t)$ & Number of susceptible vector at time $t$ & 9000 \\
\hline$I_{V}(t)$ & Number of Infected vector at time $t$ & 5000 \\
\hline$B_{H C}$ & New recruitments in susceptible human child class & 40 \\
\hline$B_{\mathrm{HF}}$ & New recruitments in susceptible human female class & 80 \\
\hline$B_{\text {нм }}$ & New recruitments in susceptible human male class & 50 \\
\hline$B_{V}$ & New recruitments in susceptible vector class & 100 \\
\hline$b$ & Vector biting rate & 50 \\
\hline$k_{\mathrm{HC}}$ & $\begin{array}{l}\text { Recovery/Treatment Rate in human child } \\
\text { (Rate at which infected human child get recovered } \\
\text { and moves towards recovered human class) }\end{array}$ & 0.15 \\
\hline$k_{\mathrm{HF}}$ & $\begin{array}{l}\text { Recovery/Treatment Rate in human female } \\
\text { (Rate at which infected human female get recovered } \\
\text { and moves towards recovered human class) }\end{array}$ & 0.35 \\
\hline$k_{\text {нм }}$ & $\begin{array}{l}\text { Recovery/Treatment Rate in human male } \\
\text { (Rate at which infected human male get recovered } \\
\text { and moves towards recovered human class) }\end{array}$ & 0.35 \\
\hline$\beta_{\mathrm{HC}}$ & Disease transmission rate in human child from vector bite & 0.5 \\
\hline$\beta_{H F}$ & Disease transmission rate in human female from vector bite & 0.25 \\
\hline$\beta_{\text {Hм }}$ & Disease transmission rate in human male from vector bite & 0.25 \\
\hline$\beta_{s}$ & Disease transmission rate in human from sexual contacts & 0.2 \\
\hline$c$ & Sexual contact rate in human & 25 \\
\hline$\beta_{v}$ & Disease transmission rate in vector & 0.4 \\
\hline$g_{F}$ & Growth rate of human from child to female & 0.35 \\
\hline$g_{M}$ & Growth rate of human from child to male & 0.35 \\
\hline$r$ & Rate of Spraying to kill vectors(insecticides) & 0.9 \\
\hline$\delta$ & Birth rate coefficient of infected new borns & 0.15 \\
\hline
\end{tabular}




\section{Continued}

\begin{tabular}{|c|c|c|}
\hline$\mu_{H}$ & Mortality rate for human & 0.14 \\
\hline$\mu_{V}$ & Mortality rate for vectors & 0.5 \\
\hline$\alpha_{H}$ & $\begin{array}{l}\text { Disease induced death rate amongst } \\
\text { human in Infection classes }\end{array}$ & 0.7 \\
\hline$\alpha_{v}$ & Disease induced death rate amongst vectors & 0.5 \\
\hline$u_{1}$ & Prevention control from vector bites & \\
\hline$u_{2}$ & $\begin{array}{l}\text { Prevention control from sexual activities } \\
\text { amongst infected humans }\end{array}$ & $\begin{array}{l}\text { Are controls and } \\
\text { taken initially }\end{array}$ \\
\hline$u_{3}$ & $\begin{array}{l}\text { Prevention control for infected human } \\
\text { female from conceiving child }\end{array}$ & $\begin{array}{c}\text { zero, will be } \\
\text { optimized to control }\end{array}$ \\
\hline$u_{4}$ & Treatment control amongst human & disease spread. \\
\hline$u_{5}$ & Spraying (insecticide) control amongst vector & \\
\hline
\end{tabular}

$$
\begin{aligned}
& d_{11}=-\frac{\left(1-u_{1}\right) b \beta_{H C}}{N_{H}} I_{V}^{*}-\mu_{H}-g_{F}-g_{M} \text {, } \\
& d_{19}=-\frac{\left(1-u_{1}\right) b \beta_{H C}}{N_{H}} S_{H C}^{*}, \quad d_{21}=g_{F}, \\
& d_{22}=-\frac{\left(1-u_{1}\right) b \beta_{H F}}{N_{H}} I_{V}^{*}-\frac{\left(1-u_{2}\right) c \beta_{S}}{N_{H}} I_{H M}^{*}-\mu_{H}, \\
& d_{26}=-\frac{\left(1-u_{2}\right) c \beta_{S}}{N_{H}} S_{H F}^{*}, \quad d_{29}=-\frac{\left(1-u_{1}\right) b \beta_{H F}}{N_{H}} S_{H F}^{*}, \\
& d_{31}=g_{M}, \quad d_{33}=-\frac{\left(1-u_{1}\right) b \beta_{H M}}{N_{H}} I_{V}^{*}-\frac{\left(1-u_{2}\right) c \beta_{S}}{N_{H}} I_{H F}^{*}-\mu_{H} \text {, } \\
& d_{35}=-\frac{\left(1-u_{2}\right) c \beta_{S}}{N_{H}} S_{H M}^{*}, \quad d_{39}=-\frac{\left(1-u_{1}\right) b \beta_{H M}}{N_{H}} I_{V}^{*}, \\
& d_{41}=-d_{39}, \quad d_{44}=-u_{4} k_{H C}-\mu_{H}-\alpha_{H C}, \quad d_{45}=\left(1-u_{3}\right) \delta \text {, } \\
& d_{49}=-d_{19}, \quad d_{52}=\frac{\left(1-u_{1}\right) b \beta_{H F}}{N_{H}} I_{V}^{*}+\frac{\left(1-u_{2}\right) c \beta_{S}}{N_{H}} I_{H M}^{*}, \\
& d_{55}=-u_{4} k_{H F}-\mu_{H}-\alpha_{H}, \quad d_{56}=-d_{26}, \quad d_{59}=-d_{29} \text {, } \\
& d_{63}=\frac{\left(1-u_{1}\right) b \beta_{H M}}{N_{H}} I_{V}^{*}+\frac{\left(1-u_{2}\right) c \beta_{S}}{N_{H}} I_{H F}^{*}, \quad d_{65}=-d_{35}, \\
& d_{66}=-u_{4} k_{H M}-\mu_{H}-\alpha_{H}, \quad d_{69}=-d_{39}, \quad d_{74}=u_{4} k_{H C}, \\
& d_{75}=u_{4} k_{H F}, d_{76}=u_{4} k_{H M}, \quad d_{77}=-\mu_{H}, \quad d_{84}=-\frac{b \beta_{V}}{N_{V}} S_{V}^{*} \text {, } \\
& d_{85}=d_{84}, \quad d_{86}=d_{84}, \quad d_{88}=-\mu_{V}-\frac{b \beta_{V}}{N_{V}}\left(I_{H c}{ }^{*}+I_{H F}{ }^{*}+I_{H M}{ }^{*}\right)-u_{5} r, \\
& d_{94}=-d_{84}, \quad d_{95}=-d_{84}, \quad d_{96}=-d_{84}, \\
& d_{98}=\frac{b \beta_{V}}{N_{V}}\left(I_{H c}{ }^{*}+I_{H F}{ }^{*}+I_{H M}{ }^{*}\right), \quad d_{99}=-\mu_{V}-\alpha_{V}-u_{5} r .
\end{aligned}
$$


With the parametric values given in Table 1 , trace $\left(J^{*}\right)<0$ and also $\operatorname{det}\left(J^{*}\right)>0$, if $R_{0}<1$. This implies that endemic equilibrium is locally asymptotically stable if $R_{0}<1$ and unstable, if $R_{0}>1$.

\section{Optimal Control}

For Zika disease an optimal control model is formulated, to derive optimal prevention from mosquito bite $u_{1}$, optimal prevention to stop pregnancy $u_{2}$ and sexual transmission $u_{3}$, optimal treatment $u_{4}$ and optimal spraying $u_{5}$ with minimal implementation cost, in order to minimise the number of infected individuals for model described by Equations (1) to (9) in the time interval $[0, T]$ with the feasible region same as given by $\Omega$ in Section 2 .

Considering the cost-functional as,

$$
\begin{aligned}
& J\left(u_{1}, u_{2}, u_{3}, u_{4}, u_{5}\right) \\
& =\int_{0}^{T}\left(w_{21}\left(S_{H C}\right)^{2}+w_{22}\left(S_{H F}\right)^{2}+w_{23}\left(S_{H M}\right)^{2}+w_{24}\left(I_{H C}\right)^{2}\right. \\
& +w_{25}\left(I_{H F}\right)^{2}+w_{26}\left(I_{H M}\right)^{2}+w_{27}\left(R_{H}\right)^{2}+w_{28}\left(S_{V}\right)^{2} \\
& \left.+w_{29}\left(I_{V}\right)^{2}+w_{11} u_{1}^{2}+w_{12} u_{2}^{2}+w_{13} u_{3}^{2}+w_{14} u_{4}^{2}+w_{15} u_{5}^{2}\right) \mathrm{d} t
\end{aligned}
$$

where, $x=\left(S_{H C}, S_{H F}, S_{H M}, I_{H C}, I_{H F}, I_{H M}, R_{H}, S_{V}, I_{V}\right), \quad u=\left(u_{1}, u_{2}, u_{3}, u_{4}, u_{5}\right)$ and $w_{i j}$ are weights to regularise the optimal control. In order minimise the disease spread using, $w_{i j}>0$, for $i=2, j=1,2, \cdots, 9$. Also to minimise the cost associated with strategies applied for preventions, treatment and spraying in a way that spread of infection can be controlled by choosing weights $w_{k m}>0$, for $k=1, m=1,2, \cdots, 5$. So, the optimal control task reads as $\min _{u} J\left(u_{1}, u_{2}, u_{3}, u_{4}, u_{5}\right)$ such that $P(x, u)=0$. Where $P(x, u)=0$ denotes the system of equations defined in (1) to (9), i.e. $\dot{x}=M(t, x, u), x(0)=x_{0}$. The optimal control $u^{*}$ can be obtained from $J\left(u_{1}^{*}, u_{2}^{*}, u_{3}^{*}, u_{4}^{*}, u_{5}^{*}\right)=\min _{u} J\left(u_{1}, u_{2}, u_{3}, u_{4}, u_{5}\right)$ such that $P(x, u)=0$.

The control set is

$$
\Gamma=\left\{\begin{array}{l}
\left(u_{1}, u_{2}, u_{3}, u_{4}, u_{5}\right) / u_{i}(t) \text { is piecewise continous on }[0, T], \\
a_{i} \leq u_{i}(t) \leq b_{i}, i=1,2,3,4,5, a_{i}, b_{i} \text { are constants in }[0,1]
\end{array}\right\}
$$

Using Lagrangian techniques for a problem along with Hamiltonian, the adjoint variable is needed to construct for the optimal control problem given by (1) to (9).

Introducing the Lagrangian to derive the optimality conditions,

$$
\begin{aligned}
L(x, u, \lambda)= & \left(w_{21}\left(S_{H C}\right)^{2}+w_{22}\left(S_{H F}\right)^{2}+w_{23}\left(S_{H M}\right)^{2}+w_{24}\left(I_{H C}\right)^{2}\right. \\
& +w_{25}\left(I_{H F}\right)^{2}+w_{26}\left(I_{H M}\right)^{2}+w_{27}\left(R_{H}\right)^{2}+w_{28}\left(S_{V}\right)^{2}+w_{29}\left(I_{V}\right)^{2} \\
& \left.+w_{11} u_{1}^{2}+w_{12} u_{2}^{2}+w_{13} u_{3}^{2}+w_{14} u_{4}^{2}+w_{15} u_{5}^{2}\right)
\end{aligned}
$$

To obtain the minimal value of the Lagrangian, defining the Hamiltonian $H$ for the control problem as, 


$$
\begin{aligned}
& H(x, u, \lambda)=w_{21}\left(S_{H C}\right)^{2}+w_{22}\left(S_{H F}\right)^{2}+w_{23}\left(S_{H M}\right)^{2}+w_{24}\left(I_{H C}\right)^{2} \\
& +w_{25}\left(I_{H F}\right)^{2}+w_{26}\left(I_{H M}\right)^{2}+w_{27}\left(R_{H}\right)^{2}+w_{28}\left(S_{V}\right)^{2} \\
& +w_{29}\left(I_{V}\right)^{2}+w_{11} u_{1}^{2}+w_{12} u_{2}^{2}+w_{13} u_{3}^{2}+w_{14} u_{4}^{2}+w_{15} u_{5}^{2} \\
& +\lambda_{1}\left(B_{H C}-\left(1-u_{1}\right) b \frac{\beta_{H C}}{N_{H}} I_{V} S_{H C}-\mu_{H} S_{H C}-\left(g_{F}+g_{M}\right) S_{H C}\right) \\
& +\lambda_{2}\left(B_{H F}-\left(\left(1-u_{1}\right) b \frac{\beta_{H F}}{N_{H}} I_{V}+\left(1-u_{2}\right) c \frac{\beta_{S}}{N_{H}} I_{H M}\right) S_{H F}+g_{F} S_{H C}-\mu_{H} S_{H F}\right) \\
& +\lambda_{3}\left(B_{H M}-\left(\left(1-u_{1}\right) b \frac{\beta_{H M}}{N_{H}} I_{V}+\left(1-u_{2}\right) c \frac{\beta_{S}}{N_{H}} I_{H F}\right) S_{H M}+g_{M} S_{H C}-\mu_{H} S_{H M}\right) \\
& +\lambda_{4}\left(\left(1-u_{1}\right) b \frac{\beta_{H C}}{N_{H}} I_{V} S_{H C}+\left(1-u_{3}\right) \delta I_{H F}-u_{4} k_{H C} I_{H C}-\left(\mu_{H}+\alpha_{H C}\right) I_{H C}\right) \\
& +\lambda_{5}\left(\left(\left(1-u_{1}\right) b \frac{\beta_{H F}}{N_{H}} I_{V}+\left(1-u_{2}\right) c \frac{\beta_{S}}{N_{H}} I_{H M}\right) S_{H F}-u_{4} k_{H F} I_{H F}-\left(\mu_{H}+\alpha_{H}\right) I_{H F}\right) \\
& +\lambda_{6}\left(\left(\left(1-u_{1}\right) b \frac{\beta_{H M}}{N_{H}} I_{V}+\left(1-u_{2}\right) c \frac{\beta_{S}}{N_{H}} I_{H F}\right) S_{H M}-u_{4} k_{H M} I_{H M}-\left(\mu_{H}+\alpha_{H}\right) I_{H M}\right) \\
& +\lambda_{7}\left(u_{4}\left(k_{H C} I_{H C}+k_{H F} I_{H F}+k_{H M} I_{H M}\right)-\mu_{H} R_{H}\right) \\
& +\lambda_{8}\left(B_{V}-\mu_{V} S_{V}-b \frac{\beta_{V}}{N_{V}}\left(I_{H C}+I_{H F}+I_{H M}\right) S_{V}-u_{5} r S_{V}\right) \\
& +\lambda_{9}\left(b \frac{\beta_{V}}{N_{V}}\left(I_{H C}+I_{H F}+I_{H M}\right) S_{V}-\left(\mu_{V}+\alpha_{V}\right) I_{V}-u_{5} r I_{V}\right)
\end{aligned}
$$

To get optimality Pontrayagin's maximum (minimum) principle, for the model is defined as follow.

If $\left(u_{1}^{*}, u_{2}^{*}, u_{3}^{*}, u_{4}^{*}, u_{5}^{*}\right)$ is optimal solution of an optimal control problem then there exists a nontrivial vector function

$$
\lambda(t)=\left(\lambda_{1}(t), \lambda_{2}(t), \lambda_{3}(t), \lambda_{4}(t), \lambda_{5}(t), \lambda_{6}(t), \lambda_{7}(t), \lambda_{8}(t), \lambda_{9}(t)\right) \quad \text { satisfying }
$$
following equations:

1) The state equation $\frac{\mathrm{d} x}{\mathrm{~d} t}=\frac{\partial H\left(t, u_{1}^{*}, u_{2}^{*}, u_{3}^{*}, u_{4}^{*}, u_{5}^{*}, \lambda(t)\right)}{\partial \lambda}$

2) The optimality condition $0=\frac{\partial H\left(t, u_{1}^{*}, u_{2}^{*}, u_{3}^{*}, u_{4}^{*}, u_{5}^{*}, \lambda(t)\right)}{\partial u}$

3) The adjoint equation $\frac{\mathrm{d} \lambda}{\mathrm{d} t}=\frac{\partial H\left(t, u_{1}^{*}, u_{2}^{*}, u_{3}^{*}, u_{4}^{*}, u_{5}^{*}, \lambda(t)\right)}{\partial x}$.

Using equations stated above for the Hamiltonian defined by (13), using state equations as given by (1) to (9), adjoint equations as

$$
\begin{gathered}
\dot{\lambda}_{1}=-2 w_{21} S_{H C}+\left(\lambda_{1}-\lambda_{4}\right)\left(\left(1-u_{1}\right) b \frac{\beta_{H C}}{N_{H}} I_{V}\right)+\lambda_{1}\left(\mu_{H}+g_{F}+g_{M}\right) \\
\dot{\lambda}_{2}=-2 w_{22} S_{H F}+\left(\lambda_{2}-\lambda_{5}\right)\left(\left(1-u_{1}\right) b \frac{\beta_{H F}}{N_{H}} I_{V}+\left(1-u_{2}\right) c \frac{\beta_{S}}{N_{H}} I_{H M}\right)+\lambda_{2}\left(\mu_{H}-g_{F}\right) \\
\dot{\lambda}_{3}=-2 w_{23} S_{H M}+\left(\lambda_{3}-\lambda_{6}\right)\left(\left(1-u_{1}\right) b \frac{\beta_{H M}}{N_{H}} I_{V}+\left(1-u_{2}\right) c \frac{\beta_{S}}{N_{H}} I_{H F}\right)+\lambda_{3}\left(\mu_{H}-g_{M}\right)
\end{gathered}
$$




$$
\begin{aligned}
& \dot{\lambda}_{4}=-2 w_{24} I_{H C}+\left(\lambda_{8}-\lambda_{9}\right)\left(\frac{b \beta_{V}}{N_{V}} S_{V}\right)+\left(\lambda_{4}-\lambda_{7}\right) u_{4} k_{H C}+\lambda_{4}\left(\mu_{H}+\alpha_{H C}\right) \\
& \dot{\lambda}_{5}=-2 w_{25} I_{H F}+\left(\lambda_{3}-\lambda_{6}\right)\left(\left(1-u_{2}\right) c \frac{\beta_{S}}{N_{H}} S_{H M}\right)+\left(\lambda_{8}-\lambda_{9}\right)\left(\frac{b \beta_{V}}{N_{V}} S_{V}\right) \\
&+\left(\lambda_{5}-\lambda_{7}\right) u_{4} k_{H F}+\lambda_{5}\left(\mu_{H}+\alpha_{H}\right)-\lambda_{5}\left(1-u_{3}\right) \delta \\
& \dot{\lambda}_{6}=-2 w_{26} I_{H F}+\left(\lambda_{2}-\lambda_{5}\right)\left(\left(1-u_{2}\right) c \frac{\beta_{S}}{N_{H}} S_{H F}\right)+\left(\lambda_{8}-\lambda_{9}\right)\left(\frac{b \beta_{V}}{N_{V}} S_{V}\right) \\
&+\left(\lambda_{6}-\lambda_{7}\right) u_{4} k_{H M}+\lambda_{6}\left(\mu_{H}+\alpha_{H}\right) \\
& \lambda_{7}=-2 w_{27} R_{H}+\mu_{H} \lambda_{7} \\
& \dot{\lambda}_{8}=-2 w_{28} S_{V}+\left(\lambda_{8}-\lambda_{9}\right)\left(\frac{b \beta_{V}}{N_{V}}\left(I_{H C}+I_{H F}+I_{H M}\right)\right)+\lambda_{8}\left(\mu_{V}+u_{5} r\right) \\
& \dot{\lambda}_{9}=-2 w_{29} I_{V}+\left(\lambda_{1}-\lambda_{4}\right)\left(\left(1-u_{1}\right) b \frac{\beta_{H C}}{N_{H}} S_{H C}\right)+\left(\lambda_{2}-\lambda_{5}\right)\left(\left(1-u_{1}\right) b \frac{\beta_{H F}}{N_{H}} S_{H F}\right) \\
&+\left(\lambda_{3}-\lambda_{6}\right)\left(\left(1-u_{1}\right) b \frac{\beta_{H M}}{N_{H}} S_{H M}\right)+\lambda_{9}\left(\mu_{V}+\alpha_{V}+u_{5} r\right)
\end{aligned}
$$

and optimality conditions are as

$$
\begin{gathered}
2 w_{11} u_{1}+\left(\lambda_{1}-\lambda_{4}\right)\left(b \frac{\beta_{H C}}{N_{H}} I_{V} S_{H C}\right)+\left(\lambda_{2}-\lambda_{5}\right)\left(b 1 \frac{\beta_{H F}}{N_{H}} I_{V} S_{H F}\right) \\
+\left(\lambda_{3}-\lambda_{6}\right)\left(b \frac{\beta_{H M}}{N_{H}} I_{V} S_{H M}\right)=0 \\
2 w_{12} u_{2}+\left(\lambda_{2}-\lambda_{5}\right) \frac{c \beta_{S}}{N_{H}} S_{H F} I_{H M}+\left(\lambda_{3}-\lambda_{6}\right) \frac{c \beta_{S}}{N_{H}} S_{H M} I_{H F}=0 \\
2 w_{13} u_{3}-\lambda_{4} \delta I_{H F}=0 \\
2 w_{14} u_{4}+\left(\lambda_{7}-\lambda_{4}\right) k_{H C} I_{H C}+\left(\lambda_{7}-\lambda_{5}\right) k_{H F} I_{H F}+\left(\lambda_{7}-\lambda_{6}\right) k_{H M} I_{H M}=0 \\
2 w_{15} u_{5}-\lambda_{8} r S_{V}-\lambda_{9} r I_{V}=0
\end{gathered}
$$

Solving optimality conditions for optimal control and the property of control space $u$ give,

$$
\begin{gathered}
u_{1}^{*}(t)=\max \left\{\operatorname { m i n } \left\{1, \frac{1}{2 w_{11}}\left[\left(\lambda_{4}-\lambda_{1}\right)\left(b \frac{\beta_{H C}}{N_{H}} I_{V} S_{H C}\right)\right.\right.\right. \\
\left.\left.\left.+\left(\lambda_{5}-\lambda_{2}\right)\left(b 1 \frac{\beta_{H F}}{N_{H}} I_{V} S_{H F}\right)+\left(\lambda_{6}-\lambda_{3}\right)\left(b \frac{\beta_{H M}}{N_{H}} I_{V} S_{H M}\right)\right]\right\}, 0\right\} \\
u_{2}^{*}(t)=\max \left\{\min \left\{1, \frac{1}{2 w_{12}}\left(\lambda_{5}-\lambda_{2}\right) \frac{c \beta_{S}}{N_{H}} S_{H F} I_{H M}+\left(\lambda_{6}-\lambda_{3}\right) \frac{c \beta_{S}}{N_{H}} S_{H M} I_{H F}\right\}, 0\right\} \\
u_{3}^{*}(t)=\max \left\{\min \left\{1, \frac{1}{2 w_{13}} \lambda_{4} \delta I_{H F}\right\}, 0\right\} \\
u_{4}^{*}(t)=\max \left\{\min \left\{1, \frac{1}{2 w_{14}}\left(\lambda_{4}-\lambda_{7}\right) k_{H C} I_{H C}+\left(\lambda_{5}-\lambda_{7}\right) k_{H F} I_{H F}+\left(\lambda_{6}-\lambda_{7}\right) k_{H M} I_{H M}\right\}, 0\right\}
\end{gathered}
$$




$$
u_{5}^{*}(t)=\max \left\{\min \left\{1, \frac{1}{2 w_{15}}\left(\lambda_{8} r S_{V}+\lambda_{9} r I_{V}\right)\right\}, 0\right\}
$$

\section{Numerical Simulations and Observations}

For parametric values given in Table 1, the basic reproduction number $R_{0}=0.4757<1, \operatorname{trace}(J)=-4.88<0, \operatorname{det}(J)=0.0014>0$, $\operatorname{trace}\left(J^{*}\right)=-4.88<0$ and $\operatorname{det}\left(J^{*}\right)=0.0041>0$ Above values verifies that non-linear system given by Equations (1) to (9) is asymptotically stable at disease free equilibrium point as well as endemic equilibrium point.

In Figure 2, global stability of human population for $R_{0}<1$ is shown. It can be seen that in the beginning susceptible human population increases but after 15 days it moves toward stability. Also, infected human population decreases initially but no prevention from vector bite, treatment, sexual activity, pregnancy, and no spraying increase infected population after 10 days. High disease induced death rate controls the infected human population.

In Figure 3, the effects of increase in prevention from mosquito bites on various infected human classes are analysed. It can be observed that prevention from vector bites in human population is an essential tool to control disease spread.

From Figure 4, it can be analysed that how the infected human population can be controlled by giving proper treatment on time. As there is no specific vaccination or treatment available for Zika disease cure, the treatment is considered as cure of symptoms of disease.

Figure 5 indicates that proper preventive steps to stop sexual transmission of disease taken at the time of disease out breaks will help to control disease spread.

From Figure 6, it can be observed that the preventions to stop pregnancy amongst infected female and effected area by ZIKV, will reduce the new child births having microcephaly and hence infected child population.

Figure 7 indicates that how the effective spraying can control infected mosquito population and hence finally spraying on mosquito will control disease spread indirectly in human population.

Thus to investigate effects of congenital transmission of ZIKV and its control
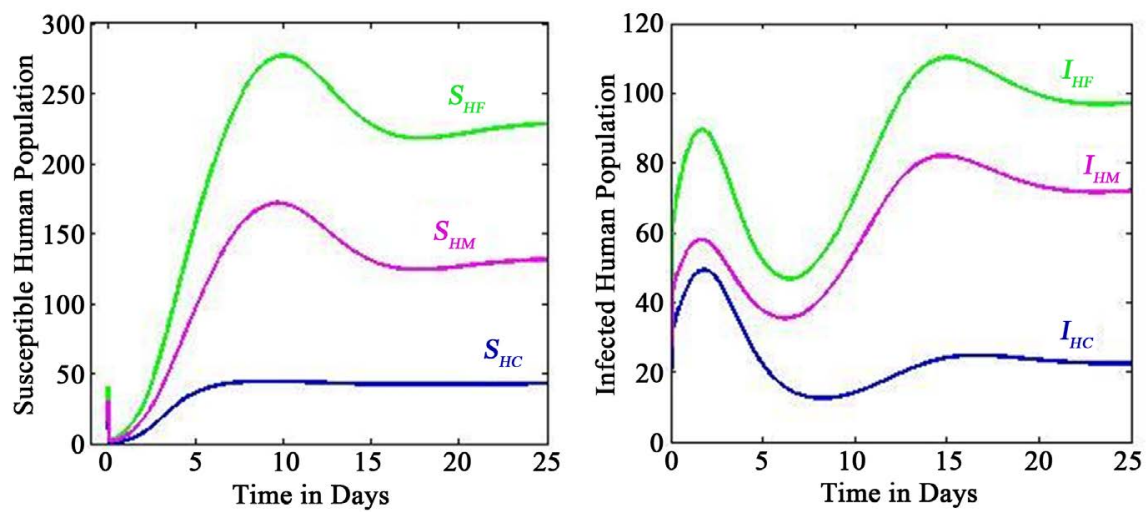

Figure 2. Global stability of human population for $R_{0}<1$. 

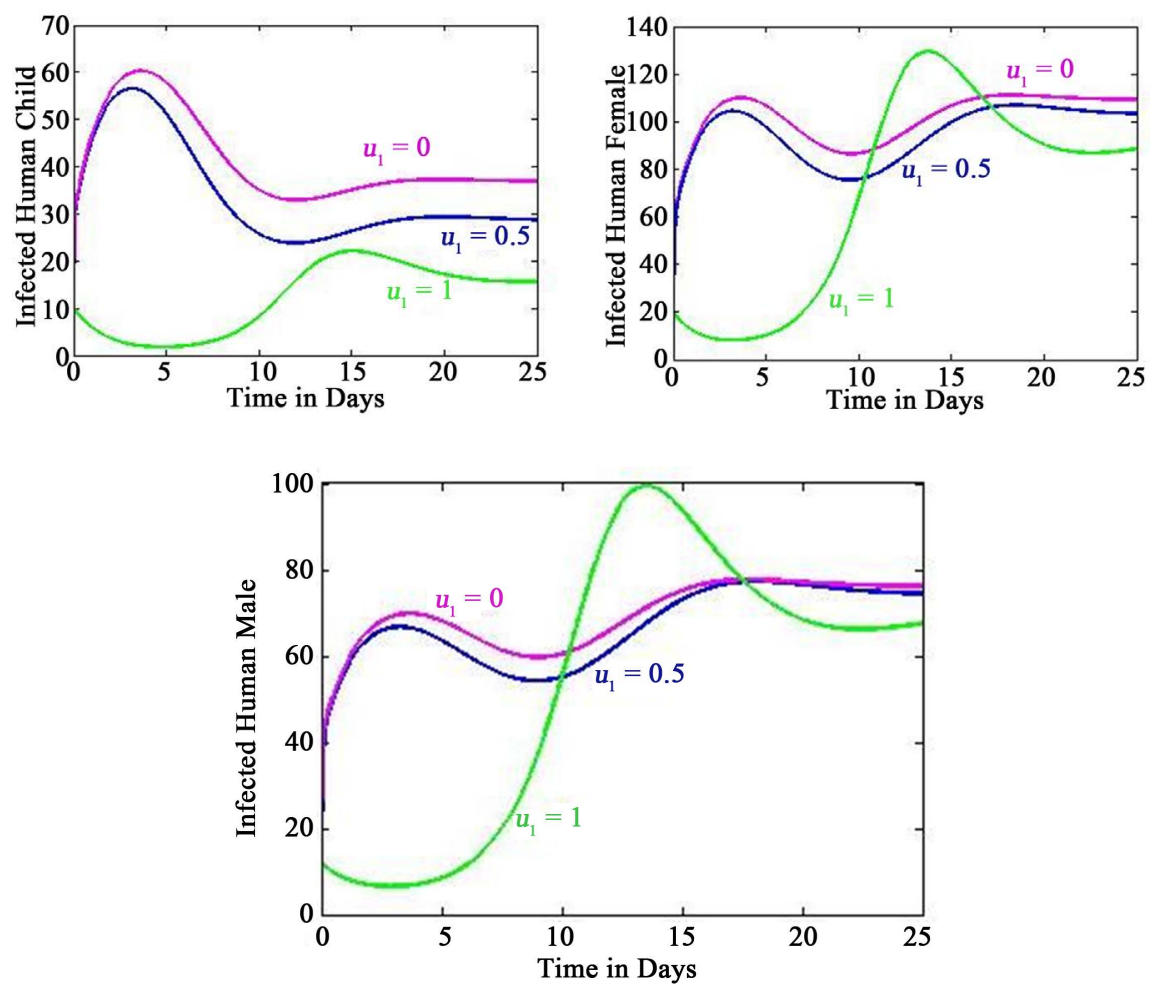

Figure 3. Effects of increase in prevention from mosquito bites on infected population.
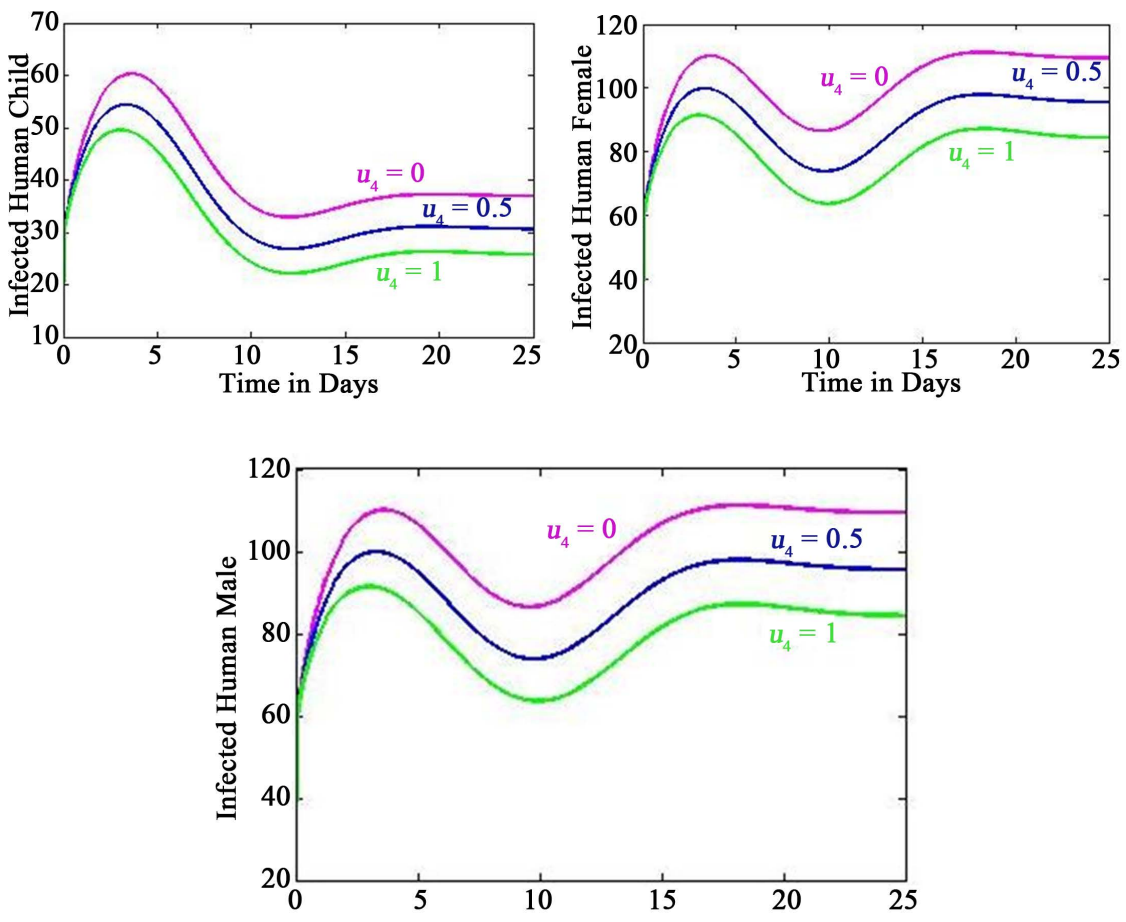

Figure 4. Effects of increase in treatment on infected population.

by spraying on mosquitoes, preventions and treatment on human population, it is required to make the total cost associated with above controls minimise using optimal policy for all controls. (Figure 8) 

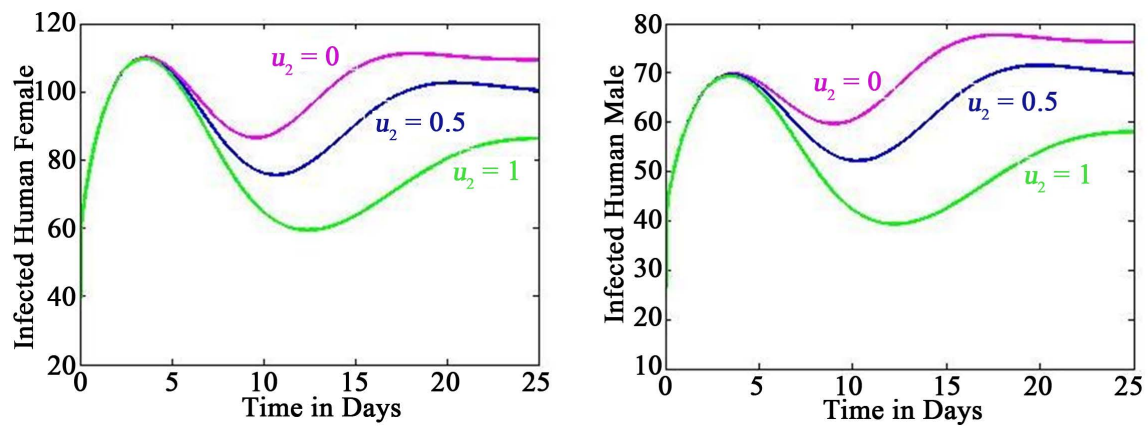

Figure 5. Effects of increase in prevention from sexual contacts on infected population.

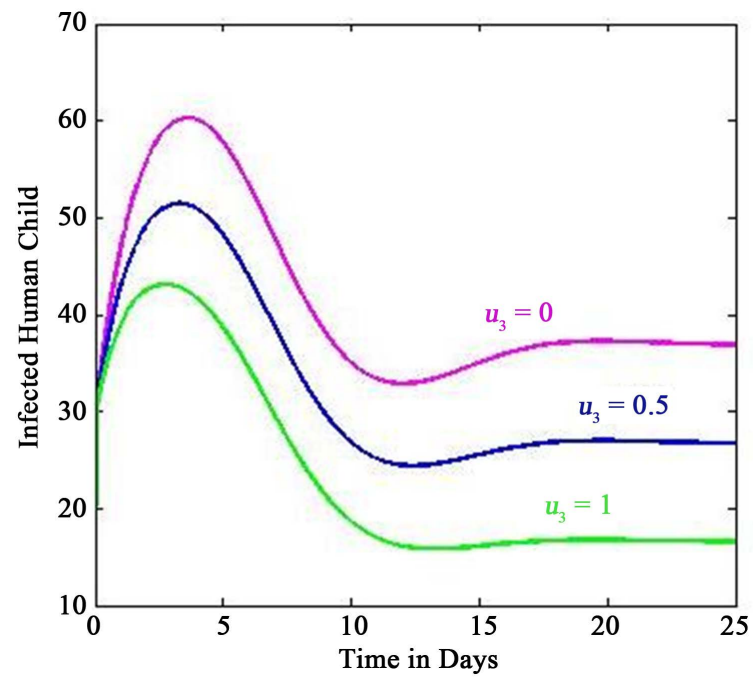

Figure 6. Effects of increase in prevention from pregnancy on infected population.

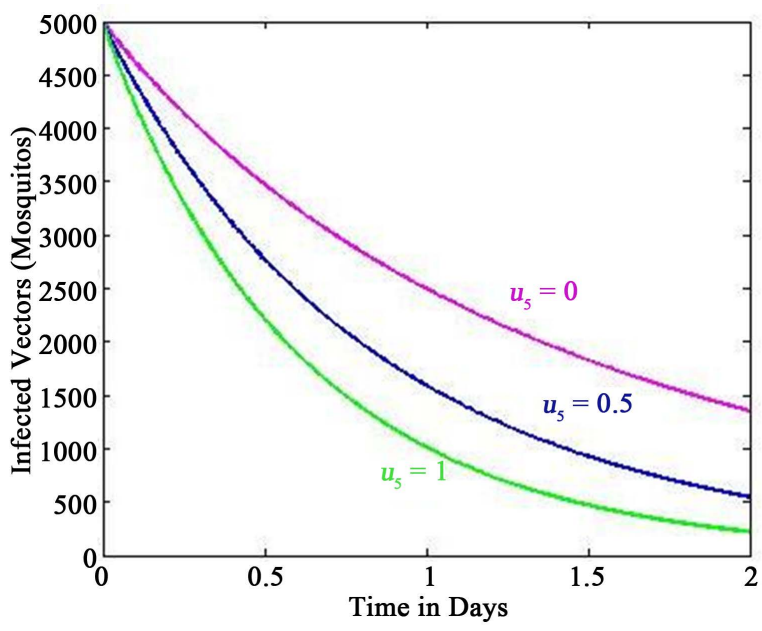

Figure 7. Effects of increase in spraying (insecticide) on infected mosquito population.

To minimise the total effective cost to control disease spray, the policy is to be designed in a way that during first week of disease outbreaks, $26 \%$ preventions on treatment and $40 \%$ prevention to stop pregnancy, $15 \%$ prevention on sexual activities amongst human, $8 \%$ spraying is required to have for $1 \%$ of prevention from vector bite. 


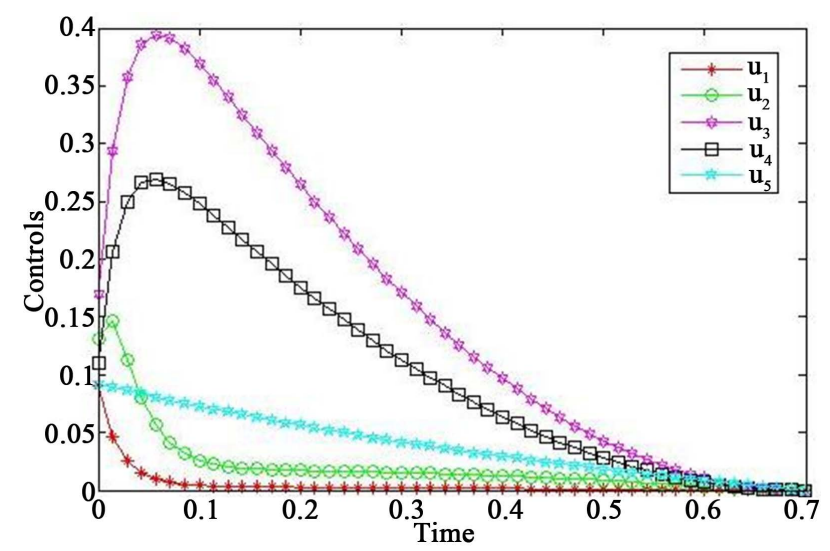

Figure 8. Controls.

\section{Conclusions}

In this paper, the spread of ZIKV considered initially as vector-borne infection and then after its spread amongst sexual partners and from mother to child is analysed. The control over spraying on vectors, prevention from vector bite, prevention during sexual activity, prevention to stop pregnancy and prevention for treatment with time incorporated. It is observed that all controls disease effectively in each compartment.

In future, the model can be studied with addition of transmission of ZIKV by blood transmission in human population.

\section{Acknowledgements}

The authors thank DST-FIST file \# MS1-097 for support to the department of Mathematics.

\section{References}

[1] Dick, G.W., Kitchen, S.F. and Haddow, A.J. (1952) Zika Virus (I). Isolations and Serological Specificity. Transaction of the Royal Society of Tropical Medicine and Hygiene, 46, 509-520. https://doi.org/10.1016/0035-9203(52)90042-4

[2] Rodrigez, A.J. (2015) Zika: The New Arbovirus Threat for Latin America. The Journal of Infection in Developing Countries, 9, 684-685.

[3] Macnamara, F.N. (1954) Zika Virus: A Report on Three Cases of Human Infection during an Epidemic of Jaundice in Nigeria. Transaction of the Royal Society of Tropical Medicine and Hygiene, 48, 139-145. https://doi.org/10.1016/0035-9203(54)90006-1

[4] Duffy, M.R., et al. (2009) Zika Virus Outbreak on Yap Island, Federated States of Micronesia. The New England Journal of Medicine, 360, 2536-2543. https://doi.org/10.1056/NEJMoa0805715

[5] Musso, D., Nilles, E.J. and Cao-Lormeau, V.M. (2014) Rapid Spread of Emerging Zika Virus in the Pacific Area. Clinical Microbiology and Infection, 20, 595-596. https://doi.org/10.1111/1469-0691.12707

[6] Pan American Health Organization (PAHO), Zika Virus Infection. http://www.paho.org/hq/index.php?option=com

[7] Foy, B.D., et al. (2011) Probable Non-Vector-Borne Transmission of Zika Virus, 
Colorado, USA. Emerging Infectious Diseases, 17, 880-882.

https://doi.org/10.3201/eid1705.101939

[8] Dallas County Health and Human Services (DCHHS), DCHHS Reports First Zika Virus Case in Dallas County Acquired Through Sexual Transmission. 2 February 2016.

https://www.dallascounty.org/department/hhs/documents/DCHHS_Zika

[9] Centers for Disease Control and Prevention (CDC). Update: Interim Guidelines for Prevention of Sexual Transmission of Zika Virus-United States, 2016. 23 February 2016. http://emergency.cdc.gov/han/han00388.asp

[10] Hills, S.L., et al. (2016) Transmission of Zika Virus through Sexual Contact with Travellers to Areas of Ongoing Transmission-Continental United States. Morbidity and Mortality Weekly Report, 65, 215-216. https://doi.org/10.15585/mmwr.mm6508e2

[11] Mansuy, J.M., et al. (2016) Zika Virus: High Infectious Viral Load in Semen, a New Sexually Transmitted Pathogen? The Lancet Infectious Diseases, 16, 405. https://doi.org/10.1016/S1473-3099(16)00138-9

[12] Toronto Star, Canada's First Case of Sexually-Transmitted Zika Virus Confirmed in Ontario. 25 April 2016.

https://www.thestar.com/news/gta/2016/04/25/first-canadian-case-of-sexually-trans mitted-zika-virus-detected-in-ontario.html

[13] Musso, D., Roche, C., Robin, E., Nhan, T., Teissier, A., Cao-Lormeau, V., et al. (2015) Potential Sexual Transmission of Zika Virus. Emerging Infectious Diseases, 21, 359-361. www.cdc.gov/eid

[14] Gatherer, D. and Kohl, A. (2015) Zika Virus: A Previously Slow Pandemic Spreads Rapidly through the Americas. Journal of General Virology, 97, 269-273. https://doi.org/10.1099/jgv.0.000381

[15] Mlakar, J., et al. (2016) Zika Virus Associated with Microcephaly. The New England Journal of Medicine, 374, 951-958. https://doi.org/10.1056/NEJMoa1600651

[16] Cauchemez, S., Besnard, M., Bompard, P., Dub, T., Guillemette-Artur, P., Eyrolle-Guignot, D., Salje, H., Van Kerkhove, M.D., Abadie, V., Garel, C., Fontanet, A. and Mallet, H.P. (2016) Association between Zika Virus and Microcephaly in French Polynesia, 2013-15: A Retrospective Study. The Lancet, 387, 2125-2132. https://doi.org/10.1016/S0140-6736(16)00651-6

[17] Cao-Lormeau, V.M., Blake, A., Mons, S., Lastère, S., Roche, C., Vanhomwegen, J., Dub, T., Baudouin, L., Teissier, A., Larre, P., Vial, A.L., Decam, C., Choumet, V., Halstead, S.K., Willison, H.J., Musset, L., Manuguerra, J.C., Despres, P., Fournier, E., Mallet, H.P., Musso, D., Fontanet, A., Neil, J. and Ghawché, F. (2016) Guillain-Barré Syndrome Outbreak Associated with Zika Virus Infection in French Polynesia: A Case Control Study. The Lancet, 387, 1531-1539.

https://doi.org/10.1016/S0140-6736(16)00562-6

[18] WHO Report.

http://www.who.int/mediacentre/news/statements/2016/1st-emergency-committeezika/en/

[19] Oliveira Melo, A.S., Malinger, G., Ximenes, R., Szejnfeld, P.O., Alves, S.S. and Bispo de Filippis, A.M. (2016) Zika Virus Intrauterine Infection Causes Fetal Brain Abnormality and Microcephaly: Tip of the Iceberg? Ultrasound in Obstetrics \& Gynecology, 47, 6-7. https://doi.org/10.1002/uog.15831

[20] Pizza, D.M.M., Loaiza, A.M., Montoya, J.F.A., Manrique, O.A., Sossa, V.A., Muñoz, C.A.A., Raigosa, S., Contreras, H.M., Contreras, I.P., Perea, M.E.C. and Enríquez, M.A. (2016) A Model for the Risk of Microcephaly Induced by the Zika Virus 
(ZIKV). Open Journal of Modelling and Simulation, 4, 109-117. https://doi.org/10.4236/ojmsi.2016.43010

[21] Bonyah, E. and Okosun, K.O. (2016) Mathematical Modeling of Zika Virus. Asian Pacific Journal of Tropical Disease, 6, 673-679.

[22] Gao, D., Lou, Y., He, D., Porco, T.C., Kuang, Y., Chowell, G. and Ruan, S. (2016) Prevention and Control of Zika as a Mosquito-Borne and Sexually Transmitted Disease: A Mathematical Modeling Analysis. Scientific Reports, 6, Article No. 28070 .

[23] Pontryagin, L.S., Boltyanskii, V.G., Gamkrelidze, R.V. and Mishchenko, E.F. (1986) The Mathematical Theory of Optimal Process. Gordon and Breach Science Publishers, New York, 4.

[24] Van den Driessche, P. and Watmough, J. (2002) Reproduction Numbers and SubThreshold Endemic Equilibria for Compartmental Models of Disease Transmission. Mathematical Biosciences, 180, 29-48.

\section{Submit or recommend next manuscript to SCIRP and we will provide best} service for you:

Accepting pre-submission inquiries through Email, Facebook, LinkedIn, Twitter, etc. A wide selection of journals (inclusive of 9 subjects, more than 200 journals)

Providing 24-hour high-quality service

User-friendly online submission system

Fair and swift peer-review system

Efficient typesetting and proofreading procedure

Display of the result of downloads and visits, as well as the number of cited articles Maximum dissemination of your research work

Submit your manuscript at: http://papersubmission.scirp.org/

Or contact am@scirp.org 